\title{
A Study of Clinical Profile in Patients with Vestibular and Non-Vestibular Vertigo
}

\section{Aditya Ranjan ${ }^{1}$, Shraddha Jain ${ }^{2}$, S.S. Chaudhari ${ }^{3}$}

Section: Healthcare

Sci. Journal Impact

Factor: 6.1 (2018)

ICV: 90.90 (2018)

(c) (3) (8)

Copyright@IJCRR

\author{
Junior resident, Department of ENT, Jawaharlal Nehru Medical College, Datta Meghe lnstitute of Medical Sciences, Sawangi Wardha, \\ Maharashtra, India; ${ }^{2}$ Professor, Department of ENT, ]awaharlal Nehru Medical College, Datta Meghe Institute of Medical Sciences, Sawangi \\ Wardha, Maharashtra, India; 3 Professor, Mechanical Engineering, Yeshwantrao Chavan College of Engineering, Nagpur, Maharashtra, lndia.
}

\section{ABSTRACT}

Background: Dizziness is the $3^{\text {rd }}$ commonest cause for presentation to a general practitioner and its causes are mainly otological followed by central, sensory and visual. Diagnosis of dizziness is majorly clinical. However, there is a lack of quality studies evaluating the clinical profile in vertigo and dizziness of different origin(vestibular and non-vestibular) and different age groups. Moreover, we have seen that a significant bulk of the patient present with "cervicogenic giddiness" but at present, there are no standard guidelines for diagnostic criteria for cervicogenic giddiness which may occur alone or associated with other types of giddiness and that treating such patients with physiotherapy can relieve and even reverse the condition.

Objectives: To study the clinical profile in patients with vestibular and non-vestibular vertigo. To study any association between cervicogenic vertigo and labyrinthine vertigo, like Benign Paroxysmal Positional Vertigo and Meniere's Disease. To study clinical features in patients with dizziness and vertigo, when multiple conditions co-exist.

Methods: In all the 96 patients of dizziness or vertigo, a pre-designed proforma will be filled, followed by a general and systemic examination with special emphasis on cardiovascular and a detailed neurological assessment. Special Clinical Tests for vertigo like Dix-Hall pike Manoeuvre, Tandem walking, Dysdiadokokinesis, tests for postural hypotension, Vertebrobasilar insufficiency, cervicogenic dizziness will be carried out.

Results: The collected data will be tabulated and statistical analysis will be done by estimating means and standard deviations for various measurements related to radiologic and other measurements.

Conclusion: This study will help the clinicians for better understanding of giddiness and differentiate between different types of giddiness in different age groups and their association with changes in the cervical spine and association with cervicogenic giddiness.

Key Words: Cervicogenic giddiness, Cervicogenic headache, dizziness, neck pain, Benign Paroxysmal positional vertigo, Meniere's disease

\section{INTRODUCTION}

Dizziness can be defined as disturbed postural awareness or vertigo, which is a perceived sense of motion ${ }^{1}$ or any illusory sense of motion without any real movement about gravity. ${ }^{2}$ Dizziness can be vestibular and non-vestibular, vestibular giddiness can be felt as a sensation of imbalance and instability and can present as pre-syncope and syncope rapid eye movement and sudden falls. Dizziness due to other causes can be felt as light-headedness, faintness or malaise which may occur due to poor central nervous system perfusion due to systemic (cardiac or metabolic causes). ${ }^{3}$
Diagnosis in patients with dizziness and vertigo depends on patients own complaints without any definitive clinical sign or investigations. Multiple conditions of dizziness may coexist, making the clinical picture more complex. ${ }^{4,5}$ Benign Paroxysmal Positional Vertigo (BPPV), followed by Vestibular neuritis and Meniere's disease, perilymph fistulas are common otological causes and can also be associated with cerebrovascular disease other diseases including migraine ${ }^{6-8}$, giddiness due to psychiatric causes, intracranial tumours. ${ }^{9,10}$. The commonest condition associated with childhood vertigo is vestibular migraine. ${ }^{11}$ In adolescents, the incidence of somatoform vertigo syndromes increases. ${ }^{12}$

\section{Corresponding Author:}

Dr. Aditya Ranjan, Junior resident, Department of ENT, Jawaharlal Nehru Medical College, Datta Meghe Institute of Medical Sciences, Sawangi Wardha, Maharashtra, India; Phone: 9304229360; Email: adityaranjan080994@gmail.com;

ISSN: 2231-2196 (Print)

Received: 20.09 .2020
ISSN: 0975-5241 (Online)

Revised: 15.10 .2020
Accepted: 04.11 .2020
Published: 30.11 .2020 
Disease of the cervical spine can also cause dizziness. Cervical spine musculature along with head position and posture are related together; in particular, there exists a balance between the cervical spine, the vestibular system, visual system and central nervous system. ${ }^{1,10}$ Cervicogenic vertigo can be presented as a separate entity but is controversial. Proprioceptive cervical vertigo is the hypothesis proposed for cervicogenic vertigo along with Barré-Lieou syndrome, rotational vertebral artery vertigo, and migraine-associated cervicogenic vertigo. ${ }^{13}$ Cervical spine degenerative changes as seen in Cervical spine spondylosis, osteoarthritis, inflammatory diseases like rheumatoid arthritis, vertebral collapse, muscle spasms, herniated discs are a major cause for abnormalities in balance, where the zygapophyseal joint are insignificant mechanical stress. The proprioceptors located in deep musculature of the cervical spine causes abnormal stimulation to the vestibular nuclei. In rotational artery vertigo, angiography-magnetic resonance, computed tomography and digital subtraction tells us about the arterial compression. ${ }^{14}$

In about $70-80 \%$ of patients with vertigo, diagnosis is mainly based on clinical examination. The duration of symptoms is different for different types of vertigo, giddiness can last for few seconds to minutes as in cases of Benign Paroxysmal Positional Vertigo, less than 24 hours as in case of Meniere's disease, giddiness lasting for days as, in cases of Vestibular Neuritis, it may be associated with Ataxia in cases of cerebrovascular diseases. However, we have found that considerable overlapping can exist between certain types of vertigo with cervicogenic vertigo-like cervicogenic vertigo and migraine or Meniere's disease. In our study, we have also found that proper physiotherapy can be an effective means for treating cervicogenic vertigo and we have seen that it benefits patient with Meniere's disease, BPPV and Migraine as well.

There are very few studies, especially from India, which have studied the clinical features in dizziness and vertigo of different etiologies, in detail. ${ }^{10,15}$ There is a dearth of data on causes and clinical features in patients of dizziness and vertigo, though it is one of the very common complaints.

A systematic review of the literature shows there is a lack of quality studies evaluating the clinical profile in vertigo and dizziness of different origin.

\section{OBJECTIVES}

1. To study the clinical profile in patients with vestibular and non-vestibular vertigo

2. To study any association between cervicogenic vertigo and labyrinthine vertigo, like Benign Paroxysmal Positional Vertigo and Meniere's Disease

3. To study clinical features in patients with dizziness and vertigo, when multiple conditions co-exist.

\section{MATERIALS AND METHODS}

This is a Prospective observational study which will be conducted in the Department of Otorhinolaryngology Acharya Vinoba Bhave Rural Hospital, Sawangi (Meghe), Wardha, attached to Jawaharlal Nehru Medical College, Sawangi (Meghe), Wardha, located in Central India comprising of the predominantly rural population of east Maharashtra including all the patients with dizziness or vertigo in the age group of more than 4 years excluding children below the age of 4 years and genetic diseases.

Sample size including 96 patients studied from august 2018 to July 2020 including patients attending Otorhinolaryngology OPD or emergency with complaints of giddiness or dizziness, or referred from General Medicine, Pediatrics, Neurology, Orthopedics and other departments for the above complaints. In all the 96 patients of dizziness or vertigo, a detailed history will be obtained according to a pre-designed proforma. This will be followed by a general and systemic examination with special emphasis on cardiovascular and a detailed neurological assessment. Special Clinical Tests for vertigo of different origin like Dix-Hall PikMan oeuvre, Tandem walking, Dysdiadokokinesis, tests for postural hypotension, Vertebrobasilar insufficiency, cervicogenic dizziness will be carried out. A detailed E.N.T evaluation will be done with the help of a Bull's lamp, head mirror, otoscope and microscope.

Apart from basic investigations, cases selected for our study will be subjected to Blood Sugar, Lipid profile, Kidney Function Tests, Thyroid profile and echocardiography, Doppler studies, Elastography of neck tissues, MR Angiography where indicated.

Audiological investigations will include Pure Tone Audiometry and Impedance Audiometry where indicated. Pure tone audiometry will be conducted in all patients as a baseline investigation for assessing the type and degree of hearing loss. It will be carried out in a sound-treated room with ALPS AD 2000 where least is $250 \mathrm{~Hz}$ and the highest is $8000 \mathrm{~Hz}$ and from $5 \mathrm{db}$ to $100 \mathrm{db}$. The frequency dial will be adjusted to $1000 \mathrm{~Hz}$. And the minimum intensity at which the subject hears will be taken as a threshold for air conduction (AC) at the frequency. Similarly, all the other frequencies $250 \mathrm{~Hz}$, $500 \mathrm{~Hz}, 2000 \mathrm{~Hz}, 4000 \mathrm{~Hz}$ and $8000 \mathrm{~Hz}$ will be tested for air conduction threshold and measured by 5 'up' and 'down' technique. The bone conduction threshold will similarly be measured. The pure tone average of the air conduction threshold will be calculated by taking the mean of the hearing thresholds at $500 \mathrm{~Hz}, 1000 \mathrm{~Hz}$ and $2000 \mathrm{~Hz}$. Then, the degree of hearing loss will be assessed, according to the WHO classification of hearing impairment. Also, the hearing thresholds at each frequency will be noted. Brain Stem Evoked Response Audiometry (BERA) will be done, where indicated. 
MRI brain (1.5 Tesla) for ruling out central vertigo, where indicated. High-Resolution Computed Tomography (CT) temporal bone and contrast-enhanced $\mathrm{CT}$ of the brain will be done in cases of suspected complicated otitis media or cases of post-traumatic vertigo.

Radiological Investigations will include X-ray cervical spine - Anteroposterior and Lateral, in all cases after inclusion in the study. MRI-cervical spine will be undertaken where indicated. Findings thus obtained will be entered in the proforma meant for the study.

\section{EXPECTED OUTCOMES/RESULTS}

The collected data will be tabulated and statistical analysis will be done by estimating means and standard deviations for various measurements related to radiologic and other measurements. Statistical analysis will be done by using descriptive and inferential statistics using Chi-square test, univariate and multivariate regression analysis by software SPSS 17.0 version, Graph Pad 6.0 version and EPI-INFO 6.0 version and $p<0.05$ will be considered as the level of significance $(\mathrm{p}<0.05)$.

\section{DISCUSSION}

This study will help the clinicians for better understanding of giddiness and differentiate between different types of giddiness in different age groups and their association with changes in the cervical spine and association with cervicogenic giddiness.

Balance and Equilibrium are maintained by sensory inputs from three different systems that are the vestibular, visual and the proprioceptive system and whereas the cerebellum co-originates and fine-tunes the motor outputs. Vertigo may be central or peripheral, vestibular or non-vestibular and symptoms have to be first differentiated from light-headedness or pre-syncope and sensation of disequilibrium or vertigo.

The most common form of dizziness is Non-specific lightheadedness. As the patient's symptom is vague and subjective, patients use terms such as giddiness or wooziness for imbalance but the patient never actually falls. Causes of nonspecific light-headedness include hyperventilation, hypoglycaemia, anaemia, head trauma, and associated with psychogenic disorders such as depression, anxiety, or phobia.

Pre-syncope is experienced as a sensation of impending fainting or altered consciousness and the patient may experience generalized weakness also. These symptoms occur while rising from a lying or sitting position. Causes are orthostatic hypotension, autonomic dysfunction due to dia- betes and cardiovascular diseases, arrhythmias, myocardial infarction, and carotid artery stenosis. Some medicines like anti-hypertensive and anti-arrhythmic drugs can also cause presyncope.

Disequilibrium is the impaired balance and gait but without abnormal head sensation. The patient has a feeling of unsteadiness. But there is no sensation of fainting. Commonly it's due to ageing. Ageing produces multi-sensory deficits. Degeneration becomes evident in the ampullae of the semi-circular ducts and the otolith organs, as well as in the vestibular nuclei and brainstem pathways. Visual acuity and proprioception also decrease with ageing and vestibular sedatives are useless in this case. A vestibular rehabilitation programme or even just a walking stick can be much helpful. Other causes of disequilibrium include peripheral neuropathy, musculoskeletal disorder, gait disorder, and Parkinson's disease. The patient who complains of disequilibrium and has a poor gait simultaneously should be referred for neurological evaluation to rule out central cause. ${ }^{16}$

Vertigo is a hallucination of movement. It can be perceived as the surrounding moving or the subject himself moving concerning the surrounding. It indicates lesion at the vestibular nuclei. Few articles focussed on related aspects of this issue were reported. ${ }^{17-20}$ Jain et al. reported a study on Cervicogenic Vertigo. ${ }^{21}$

\section{ACKNOWLEDGMENT}

Authors acknowledge the immense help received from the scholars whose articles are cited and included in references to this manuscript. The authors are also grateful to authors / editors / publishers of all those articles, journals, and books from which the literature for this article has been reviewed and discussed.

\section{Conflict of Interest: Nil}

\section{Source of Funding: Nil}

\section{REFERENCES}

1. Burt CW, Schappert SM. Ambulatory care visits to physician offices, hospital outpatient departments, and emergency departments; the United States, 1999-2000: data from the National Health Care Survey.

2. Committee on Hearing and Equilibrium. Committee on Hearing and Equilibrium guidelines for the diagnosis and evaluation of therapy in Meniere's disease. Otolaryngol Head Neck Surg 1995;113(3):181-5.

3. Bittar RS, Oiticica J, Bottino MA, Ganança FF, Dimitrov R. Population epidemiological study on the prevalence of dizziness in the city of Sao Paulo. Braz J Otorhinolaryngol 2013 Nov;79(6):688-98.

4. Treleaven J. Sensorimotor disturbances in neck disorders affecting postural stability, head and eye movement control. Manual Therapy 2008;13(1):2-11. 
5. Brandt T. Cervical vertigo-reality or fiction? Audiol Neurotol 1996;1(4):187-96.

6. Yacovino DA. Cervical vertigo: myths, facts, and scientific evidence. Neurologia (Barcelona, Spain). 2012 Sep 13.

7. Reiley AS, Vickory FM, Funderburg SE, Cesario RA, Clendaniel RA. How to diagnose cervicogenic dizziness. Arch Physiother 2017;7(1):12.

8. Bjorne A, Berven A, Agerberg G. Cervical signs and symptoms in patients with Meniere's disease: a controlled study. CRANIO 1998;16(3):194-202.

9. Bjorne A, Agerberg G. Symptom relief after treatment of temporomandibular and cervical spine disorders in patients with Meniere's disease: a three-year follow-up. CRANIO. 2003;21(1):50-60.

10. Elies W. Cervical vertebra-induced hearing and equilibrium disorders. Rec Clin Aspects 1984;32(12):485-93.

11. Bansal M. Common causes of vertigo and dizziness in Gujarat. Int J Clin Trials 2016 Oct;3(4):250-3.

12. Davitt M, Delvecchio MT, Aronoff SC. The differential diagnosis of vertigo in children: a systematic review of 2726 cases. Pediatr Emerge Care 2020;36(8):368-71.

13. Jahn K, Langhagen T, Schroeder AS, Heinen F. Vertigo and dizziness in childhood-update on diagnosis and treatment. Neuropediatrics 2011;42(4):129-34.

14. Li Y, Peng B. Pathogenesis, Diagnosis, and Treatment of Cervical Vertigo. Pain Physician 2015; 18: E583-E595.

15. Attry S, Gupta VK, Marwah K, Bhargav S, Gupta E, Vashisth N. Cervical vertigo-pathophysiology and management: an update. IOSR J Dental Med Sci 2016;1(15):98-107.
16. Kameswaran M, Pujari S, Singh J, Basumatary LJ, Sarda K, Pore R. Clinicoetiological pattern and pharmacotherapy practices in patients with new onset vertigo: findings from a prospective multicenter registry in India. Int J Otorhinolaryngol Head Neck Surg 2017 Apr;3(2):404.

17. Jain S, Deshmukh PT, Lakhotia P, Kalambe S, Chandravanshi D, Khatri M. Anatomical study of the facial recess with implications in round window visibility for cochlear implantation: personal observations and review of the literature. Int Arch Otorhinolaryngol 2019;23(3):281-91.

18. Jain S, Gaurkar S, Deshmukh PT, Khatri M, Kalambe S, Lakhotia $P$, et al. Applied anatomy of the round window and adjacent structures of tympanum related to cochlear implantation. Braz J Otorhinolaryngol 2019;85(4):435-46.

19. Jain S, Singh P, Methwani D, Kalambe S. Role of Eustachian Dysfunction and Primary Sclerotic Mastoid Pneumatisation Pattern in Aetiology of Squamous Chronic Otitis Media: A Correlative Study. Indian J Otolaryngol Head Neck Surg 2019;71(2):1190-6.

20. Singh P, Jain S, Methwani D, Kalambe S, Chandravanshi D, Gaurkar S, Deshmukh PT. Study of Correlation of Pre-Operative Findings with Intra-Operative Ossicular Status in Patients with Chronic Otitis Media. Iran J Otorhinolaryngol 2018 Sep;30(100):273.

21. Jain S, Jungade S, Ranjan A, Singh P, Panicker A, Singh C, et al. Revisiting Meniere's Disease as Cervicogenic Endolymphatic Hydrops" and Other Vestibular and Cervicogenic Vertigo as Spectrum of the Same Disease: A Novel Concept. Indian J Otolaryngol Head Neck Surg 2020; 22:1-6. 